\title{
0158 ESTIMATING THE BURDEN OF INJURIES IN MOAMBIQUE
}

J P Abraham*, K Bhalla, K Bartolomeos, C de Silva, F Zacharias, E Zacharias Correspondence: Department of Global Health \& Population, Harvard School of Public Health, Harvard University, 718 Huntington Avenue, 1st Floor, Boston, MA 02115-5924, USA

10.1136/ip.2010.029215.158

In 2002, the Global Burden of Disease (GBD) study estimated that sub-Saharan Africa had the highest incidence rate of injuries worldwide. Using our established methodology we triangulate a national snapshot for Moambique from multiple data sources (including hospitals, health surveys, death registers, crime reports, etc). We conducted an environmental scan of all existing data sources that can inform estimates of the incidence of fatal and non-fatal injuries. From these, we constructed estimates of the national burden of injuries by harnessing the strength of each source and accounting for biases in data.

\section{Data sources}

Estimating fatal injuries:

-Urban mortality data from Maputo City Mortuary (1994-2003)

-Rural mortality surveillance data (verbal autopsy) from Manhia DSS site (1999-2002).

Estimating non-fatal injuries:

-Moambique DHS 2003 Injury Module (2003)

-Maputo Central Hospital data (2001-2002).

Building estimates

The data were mapped to corresponding GBD external cause and nature groupings. When applicable, the data were disaggregated to compute age-sex-injury rates. The rates were then applied to urban and rural population estimates, respectively. Demographic and health surveys data were used to develop national injury envelopes. External cause to nature of injury mappings were developed using the hospital data. Using these methods, it was estimated that there were $~ 8556$ urban deaths and 13717 rural deaths for a total estimated injury deaths in 2005 of 22 273. The results were then compared with police figures and GBD estimates. 